\title{
Creating Visceral Personal and Social Interactions in Mediated Spaces
}

\author{
Kelly Dobson, danah boyd, Wendy $\mathrm{Ju}^{+}$, Judith Donath, Hiroshi Ishii ${ }^{++}$ \\ MIT Media Lab, 20 Ames Street, Cambridge, MA 02139 \\ Sociable Media, Personal Information Architecture ${ }^{+}$, Tangible Media ${ }^{++}$ \\ \{monster, danah, wendyju, judith, ishii $\} @$ media.mit.edu
}

\begin{abstract}
We introduce vibration and temperature as visceral modes to aid intuitive social perception in networked interaction. We describe two implementations of these ideas for mediated systems - VibroBod for interpersonal communication and What's Shaking for newsgroup navigation.
\end{abstract}

\section{Keywords}

Social intuition, tangible media, vibration, temperature

\section{INTRODUCTION}

Existing communication devices (such as telephones) and applications (such as chat rooms, news groups, or e-mail discussions) allow users to talk, verbally or textually, in a shared social space. Newer visual media are beginning to enable people to see what digital social spaces are like. We are interested in using other sensory modes to allow participants to viscerally feel what these spaces are like, and to convey their feelings to other people. We focus on how vibration and temperature convey such feelings.

Form plays a critical role in the design of tangible media that utilize vibration and warmth. The form should be a function of how the device is to be held or worn, where the sensations are to be felt on the body, and how strong the sensations should feel; these factors strongly influence how the warmth and vibrations are mapped to meaning. We have pursued two projects that utilize heat and vibration for applications of computer-mediated social spaces, with different forms and mappings that fit their respective applications. In the following descriptions of these prototype projects, VibroBod and What's Shaking, we discuss applications, implementation and future directions of how to use vibration and temperature to improve social intuition in mediated interaction.

\section{BACKGROUND}

The use of tangible media to convey sensations such as touch, motion[1], scent or warmth[2] has been explored, facilitating more intimate telepresence. We are interested, instead, in how warmth and vibration can be used to create emotional response. Warmth and vibration map naturally into the realm of human interactions. Warmth implies "touch" and body heat while vibration implies the "buzz" of activity. People correlate the combination of heat and lowlevel motion with human contact.

Temperature and vibration are too inexact to convey explicit messages or complex information, but that ambiguity works well for communicating "fuzzier" degrees of atmosphere, ambiance, or affect. The sensation of vibration or warmth triggers gut-instinct responses, which work well for conveying ambient information of for communicating urgency.

\section{VIBROBOD}

VibroBod allows two users to communicate feeling; hand gestures and vocalizations made by one user convey emotional content to the other user. VibroBods rest on the laps of individuals having personal conversations via phone, chat or instant messaging, to amplify moods or tones that may otherwise be lost. The design intent was to facilitate awareness, empathy, and emotional influence across mediated channels.

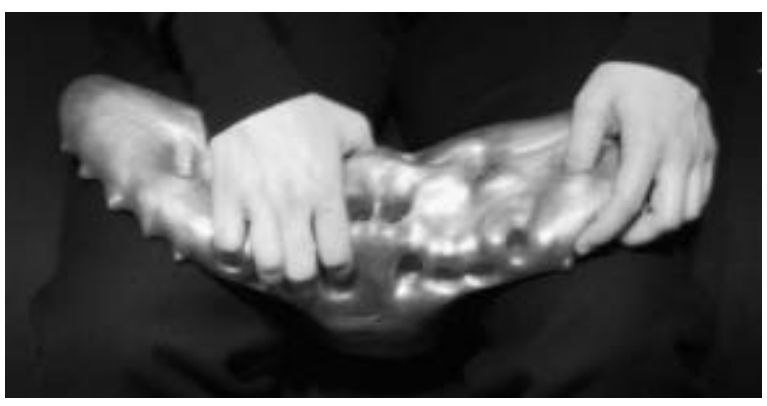

Fig 1. VibroBod prototype

VibroBod's rubber body contains force-sensitive resistors and microphones, capturing hand position and pressure as well as vocalizations. VibroBod has various finger holds to afford different gestural positions which correspond with different emotional states; more anxious finger grips, for example, are deep holes set so that both hands and individual fingers are contracted close together, whereas relaxed finger grips accommodate arms and hands much further apart in more restful positions. The force of a user's grip produces different pressures on the force sensitive 
resistors, which, combined with vocal inputs, affect different frequencies and vibration patterns inducing distinct psychological states[2]. The VibroBod grows warmer with use, and that warmth, combined with the hand holding, body hugging form, gives users the sense of holding and of being held.

In informal critiques with fifteen students and faculty, we found that the VibroBod indeed provoked strong reactions. At first, people were hesitant to place their fingers in the holes and were alarmed by the vibrations. As users learned how their actions were affecting the VibroBods' behavior, they became very involved and attentive in experiential discovery and in trying to evoke particular vibration patterns that seemed like accurate representations of how they were feeling.

In interacting through VibroBod, one user complained of feeling funny or dizzy. In the future, we would like to further research how varying vibration patterns correspond to different states, so as to prevent such instances. Also, it might be desirable to build in a smoother range of possible holds and states by refining the design of the finger holds. Most users found VibroBod to facilitate meaningful experiences and did not want to stop using the system.

\section{WHAT'S SHAKING}

What's Shaking aids in the navigation of online newsgroups by providing visceral channels of social dynamics perception. Existing graphic visualizations[4] illustrate quantitative differences between groups with similar titles by graphically conveying statistical information; our device is designed to add qualitative representations of the different dynamics and styles of interaction within groups.

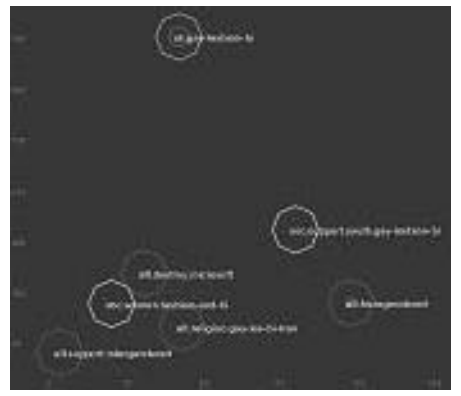

Fig 2. What's Shaking visualization

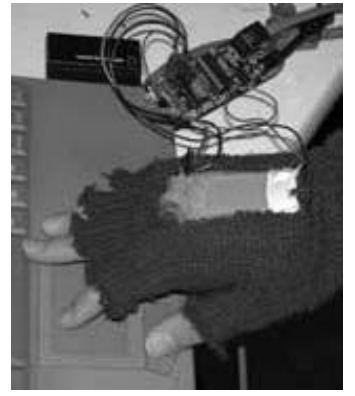

Fig 3. What's Shaking glove
The What's Shaking prototype is a glove instrumented with a pager motor and a Peltier junction, connected via a serial port to the host computer. As a user navigates Usenet newsgroups, we map the normalized average number of posts per person and the number of unique people posting in one month to the vibration and temperature of the glove, respectively. In this way, users sense populous groups as warm and active groups as vibrant.

To informally test our system, we asked twelve fellow students to use our prototype to explore newsgroups related to gender and identity. Users were quick to correlate varying degrees of warmth and vibration to meaning, with discernable and believable variations between similarly titled groups. Newsgroups with comparable warmth and vibration, such as support groups, gave users a sense of welcome. In contrast, users were surprised by groups in which temperature and vibration were seemingly mismatched, such as spam groups which have high volume but lack ongoing participation. Such uncommon couplings were instantly recognized as anomalous, suggesting that people's innate understandings of heat and vibration may eliminate any need for training or explanation if the mappings are designed correctly.

When using What's Shaking, many users were alarmed the first time they felt the warming and shaking sensations, and wanted to have an easier means of escaping these startling sensations that they could not control. This concern about form has inspired us to experiment with other forms, such as wands, pucks or balls. We would also to map these devices to synchronous on-line communities, particularly chat. Because synchronous communities change in population and tone depending on the time of day, a visceral interface may help users find an appropriate group at a given time.

\section{SUMMARY}

In both VibroBod and What's Shaking, users were able to perceive social information viscerally in mediated communication through an intuitive understanding of vibration and temperature. In VibroBod, vibration helped users convey presence, emotion, and empathy. In What's Shaking, users were able to use vibration and temperature to get a viscerally driven understanding of social interaction in newsgroups. Our prototypes demonstrate that vibration and temperature are good tools for evoking an immediate and emotional reaction, and for providing qualitative ambient social information during mediated interaction.

\section{ACKNOWLEDGMENTS}

We would like to thank the Sociable Media, Tangible Media, and Personal Information Architecture Groups at the MIT Media Lab for their support, ideas and encouragement. In addition, many thanks to Hyun-Yeul Lee for her help sparking these ideas.

\section{REFERENCES}

1. Brave, S., Daley, A., "inTouch: A Medium for Haptic Interpersonal Communication,” CHI '97, 363-364.

2. Strong, R., Gaver, W., "Feather, Scent and Shaker: Supporting Simple Intimacy," Proc. CSCW'96, 29-30.

3. Berglund, B., Hassmen, P., "Sources and effects of low frequency noise," J. Acoust. Soc. Am., 99(5) 2985-3002, May 1996.

4. Smith, M., Netscan: Measuring and Mapping the Social Structure of Usenet, Presented at the $17^{\text {th }}$ Annual International Sunbelt Social Network Conference, 1997. 